\title{
Muscle strength, voluntary activation, twitch properties, and endurance in patients with fibromyalgia
}

\author{
Jesper Nørregaard, Per Martin Bülow, Bente Danneskiold-Samsøe
}

\begin{abstract}
Previous studies have shown decreased voluntary muscle strength and endurance in patients with fibromyalgia. The aim of this study was to determine to what extent this is due to lack of exertion. The twitch interpolation technique was used to determine the degree of central activation and estimate the "true" quadriceps muscle strength in patients with fibromyalgia and age and sex matched controls. Subjects hereafter performed an endurance test consisting of repetitive contractions at $50 \%$ of estimated "true" muscle strength of four seconds duration followed by a six second rest until exhaustion, or maximally for $\mathbf{4 0}$ minutes. Twitch decline and increases in mean rectified EMG were used as objective markers of fatigue. The estimated "true" muscle strength was 82 (SD 26) $\mathrm{Nm}$ in 20 patients with fibromyalgia compared with $133 \mathrm{Nm}$ (SD 28) $\mathrm{Nm}$ in the 21 controls $(p<0.001)$. The "true" muscle strength per $\mathrm{cm}^{2}$ midthigh cross sectional area was lower $0.50(\mathrm{SD} 0.15) \mathrm{Nm} / \mathrm{cm}^{2}$ in the patients compared with 0.74 (SD $0.15) \mathrm{Nm} / \mathrm{cm}^{2}$ in the controls $(\mathrm{p}<0.001)$. The decline over time in twitch sizes was similar in the two groups. The mean rectified EMG signal at a fixed force level of $50 \%$ of "true" muscle strength increased similarly in the two groups. Relaxation rates and contraction rates also increased equally in the two groups. In conclusion, a reduction of the estimated muscle strength per area unit of about $35 \%$ was found in the patients with fibromyalgia. This might be secondary to physical inactivity or neuroendocrine factors. No differences in changes in the neurophysiological indices associated with fatigue were found between the two groups.
\end{abstract}

(F Neurol Neurosurg Psychiatry 1994;57:1106-1111)

Department of Rheumatology, Frederiksberg Hospital, Denmark J Nørregaard P M Bülow

B Danneskiold-Samsøe

B Danneskiold-S Dr Jesper Norrega: Dr Jesper Norregaard Bomlærkevej 14, 2970 Horsholm, Denmark.

Received 5 May 1993 and in final revised form 6 January 1994. Accepted 11 February 1994

Fibromyalgia has recently gained acceptance as a World Health Organisation (WHO) diagnosis. Classification criteria for fibromyalgia have been developed by the American College of Rheumatology (ACR) after a large multicentre study. ${ }^{1}$ These criteria consist of symptoms of generalised pain of at least three months duration, and pain by palpation with a pressure of $4 \mathrm{~kg}$ at more than 11 out of 18 specified tender point locations. In contrast with previous criteria, these do not include common symptoms of sleep disturbances and fatigue, and they define the condition primarily as a pain syndrome. The usage of a diagnosis of fibromyalgia has, however, been the subject of much controversy due to symptoms being suspected of having a psychical aetiology. Muscle biopsy studies of patients with fibromyalgia have shown no specific abnormalities, but a much higher occurrence of unspecific degenerative changes. ${ }^{23} \mathrm{~A}$ decreased content of energy rich phosphates ${ }^{4}$ and non-homogeneous oxygen saturation ${ }^{5}$ have also been reported in the muscles of patients with fibromyalgia, possibly indicating an abnormality of the muscles.

Besides chronic generalised pain, patients with fibromyalgia often complain of muscle fatigue during exercise and of worsening in the symptoms after exercise. Previous studies have shown decreased voluntary muscular strength and endurance in patients with fibromyalgia. ${ }^{6-8}$

The degree of central activation (neural motor drive) can be determined by the twitch interpolation technique ${ }^{9-11}$; thus discriminating central from peripheral causes of muscle strength reduction and low endurance.

Force response to electrical stimulation during fatigue is known to decline. The force decline during fatigue and recovery depends on the stimulation frequency. ${ }^{12}{ }^{13} \mathrm{~W}$ ith intermittent submaximal contractions low frequency fatigue rapidly occurs and recovers slowly in contrast with high frequency fatigue, which develops slowly and recovers quickly. ${ }^{12} 13$ It has been suggested that the low frequency fatigue is because of an impaired excitationcontraction coupling, whereas the high frequency fatigue might be primarily due to a decline in energy rich phosphates. ${ }^{13}$ During fatigue the response to single stimuli (twitch) declines in a manner similar to the low frequency stimulation response. ${ }^{13}$ Increases in the EMG amplitude during fatigue correlate primarily with the high frequency fatigue. ${ }^{14}$

The aim of this study was to compare the estimated "true" muscle strength of the quadriceps muscle of patients with fibromyalgia with a matched healthy, sedentary control group, and to relate the muscle strength to the cross sectional area of the thigh muscle. We also wanted to determine if abnormal changes in established objective physiological indices associated with fatigue could be found during an endurance test. 
Methods

SUBJECTS

From our register of patients with fibromyalgia, 28 female patients aged between 26 and 69 years were randomly selected for the study. Only women were chosen as they represent about $85 \%$ of patients with fibromyalgia. Patients with a poor record of cooperation in muscle strength measurements were not invited to participate in the study. One declined to participate, two were excluded as they were receiving medication influencing the cardiovascular system. The patients all fulfilled the ACR criteria. Blood tests taken to exclude the symptoms of fibromyalgia being caused by other diseases included erythrocyte sedimentation rate, blood cell count, thyroid hormone concentrations, creatine kinase, myoglobin, calcium, rheumatoid factor, antinuclear antibodies, and hepatic enzymes.

Twenty one healthy, age matched women were selected as a control group. Sedentary women were recruited through an advertisement at the hospital. They were not allowed to receive any medication affecting the cardiovascular system or to perform any type of regular sports. Subjects walking more than $3 \mathrm{~km}$ a day or bicycling more than $5 \mathrm{~km}$ a day were excluded.

PHYSICAL ACTIVITY LEVEL

We used a modified version of a previously used recall ${ }^{15}$ to review the number of hours spent each day on different physical activity levels: (a) light activity (slow walking, shopping, busy office work, slow bicycling); (b) moderate physical activity (fast walking, heavy cleaning or house work, moderate bicycling, easy sports); (c) Heavy physical activity (running, fast bicycling, heavy work). These three levels correspond roughly to an energy consumption of two, four, and six times the basic metabolic rate. ${ }^{15}$ These values were summed and the resting time added to get an estimated daily energy consumption in metabolic equivalents (METs). (The coefficient of variation of the daily energy consumption was $10 \%$ in 10 subjects interviewed twice within two to three weeks (unpublished results)).

MEASUREMENTS OF CROSS SECTIONAL AREA The circumference of the left thigh was measured with a tape at "midthigh"- that is, the precise midpoint between the patella and the anterior superior iliac spine. The distance from the skin to the muscle (subcutaneous fat thickness) was measured with real time ultrasound imaging (Aloka Sect Scan SSD 720) ${ }^{1617}$ at the anterior position of the thigh corresponding to the midthigh site. The fat free area was calculated from an assumption of the same fat thickness in the whole circumference.

\section{ISOMETRIC TWITCH INTERPOLATION} TECHNIQUE

Muscle strength

Isometric torque of the left quadriceps muscle was measured in a sitting position and a $90^{\circ}$ flexion in the knee joint on a Kin-Com isoki- netic dynamometer. Velcro straps were applied tightly across the pelvis and distal thigh. All measurements were corrected for the weight of the distal lower limb by subtraction of the resting value. The seat and back supports were stiff to reduce the loss of twitch force due to viscoelastic properties in the support. The optimal stiffness of the supports and fixation was a compromise between the optimal conditions for the twitch measurements and the comfort of the subject, as an uncomfortable support might reduce the central activation and thus the muscle force. The subjects were given visual feedback of the force applied in all muscle strength measurements.

\section{Percutaneous electrical stimulation}

Two pad electrodes $(5 \times 9 \mathrm{~cm})$ were placed one third and two thirds respectively of the distance between the anterior superior iliac spine and the upper border of the patellas. Electrical stimulation was given using a stimulator (15E05, 15E07 stimulator output unit, DISA, Copenhagen, Denmark). A train of six square formed stimuli $(100 \mathrm{~mA}, 0.3 \mathrm{~ms})$ was given over a three second period.

\section{EMG registration}

Surface EMG electrodes were placed over the vastus lateralis, the vastus medialis, and an indifferent electrode was placed over the lateral condyle. The EMG signal was rectified and filtered (KCEMG 5070) and stored together with the force signal for later analysis. From the stimulus artifact the time of stimulation could be identified. The EMG values of the three seconds after the first stimulation was averaged excluding the $250 \mathrm{~ms}$ after each stimulation.

\section{Measurements of twitch size}

The torque signal was $A / D$ converted with a sampling frequency of $100 \mathrm{~Hz}$ and the force resolution was $1 \mathrm{~N}$. In all measurements the subject retained the force at a level specified by a horizontal line on the screen (the measuring level). This was kept as stable as possible for at least four seconds while stimulation was given. An averaged twitch was calculated for each force level. Just before each twitch measurement the subject produced a maximal voluntary contraction for five seconds to ensure that a maximal potentiation level was achieved.

\section{Determination of "true" muscle strength}

Applying the twitch interpolation technique to a large muscle group such as the quadriceps muscle, the relation between twitch amplitude and force is not linear at forces lower than $20-25 \%$ of maximum, due to a viscoelastic force loss in the muscles (fig 1). ${ }^{18}$ Thus twitch amplitudes measured at forces lower than $20-25 \%$ cannot be used in calculation of the "true" muscle strength. Therefore, subjects were instructed to perform contractions at $100,25,50$, and $75 \%$ of their maximal voluntary strength. Electrical stimulations were given at all contraction levels. The estimated 
Figure 1 Relation between additional torque obtained by stimulation (twitch amplitude) and force level from the quadriceps muscle in two normal subjects. The voluntary contraction is shown as percentage of maximum voluntary torque and the twitch as a percentage of maximum twitch.

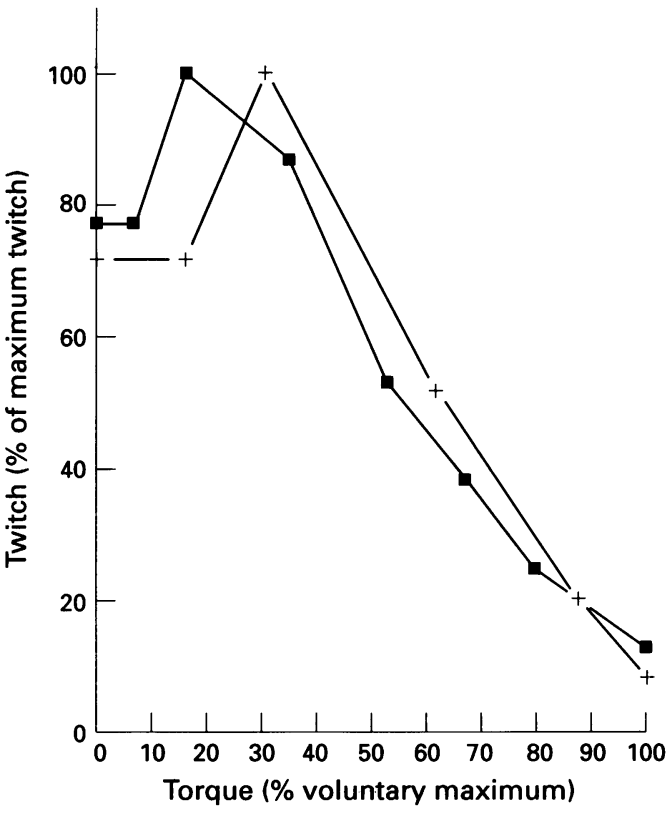

"true" muscle strength was calculated from linear extrapolation of the twitch/force relation to the zero level of twitch, corresponding to a "true" maximal voluntary contraction. .11 Twitches at the lowest contraction levels, which in a visual examination differed from a straight line, were excluded from the analysis (fig 1). The relation between torque and twitch amplitude was similarly linear in the two groups.

In a methodological study we found that if subjects maximally apply only $75 \%$ of their maximal strength, the "true" muscle strength was underestimated by a mean 3\% compared with estimation from measurement when subjects performed maximal effort. ${ }^{18} \mathrm{~A}$ more imprecise estimation and a mean underestimation of $8 \%$ was seen in subjects maximally applying $50 \%$ of maximum strength. The underestimation was due to a slight curvilinear shape of the twitch/force relation, which has also been reported earlier. ${ }^{11}$ In a later study we found a coefficient of variation of $11 \%$ for repeated determination of the "true" muscle strength in 15 patients with fibromyalgia performing at least $50 \%$ of maximum, and $6 \%$ in 13 healthy subjects.

The estimated "true" muscle strength was related to calculated fat free thigh area.

ENDURANCE PROTOCOL

The endurance protocol included repeated contractions at $50 \%$ of estimated "true" maximal strength for four seconds followed by a six second rest period. This was repeated until exhaustion or maximally for $\mathbf{4 0}$ minutes. At the time points of $0,2,4,7,10,15,20,25$, 30,35 , and 40 minutes twitch sizes were measured at $50 \%$ of the initially estimated true muscle strength (after a maximal contraction). Superimposed twitch on a submaximal force level was chosen because the twitch amplitude in the resting muscle is much influenced by the viscoelastic properties of the quadriceps muscle (see earlier) and as previous studies have reported a linear decline of twitch amplitude to zero if measured at a submaximal level..$^{12}$ In a methodological study we found that twitch amplitude measured at a force level of $50 \%$ of estimated maximum, declined exponentially during time (unpublished results):

$$
\text { Twitch }_{\mathrm{t}}=\text { Twitch }_{\mathrm{t}=0} \times \mathrm{e}^{-\mathrm{kt}} \text {. }
$$

In a few subjects who did not perform maximally from the start, an initial incline in twitch size or a constant twitch size occurred during the first few minutes. This was due to insufficient potentiation from the start. Subsequently the values declined exponentially, which indicated that maximal potentiation was achieved after a few minutes of the endurance protocol. These initial twitch values were therefore excluded from the analysis.

Relative mean EMG amplitude was calculated as actual EMG amplitude divided by the minimal EMG amplitude during the endurance test. A normalised contraction rate was calculated as the maximal incline during 0.01 seconds divided by the twitch size (for the averaged twitch). ${ }^{20} \mathrm{~A}$ normalised relaxation rate was calculated as the maximal decline during 0.01 seconds divided by the twitch size (for the averaged twitch). During the test the degree of perceived exertion was scored from 0 to 10 with a modified Borg scale. ${ }^{21}$

\section{Statistical analysis}

Data are presented as mean (SD) and compared by two sample $t$ test unless otherwise stated. Correlations were performed as Pearson's product-moment coefficient of correlation $(R)$. The endurance time and exponential constant are presented as median and interquartile range and compared by the unpaired rank sum test (Mann-Whitney) due to non-normal distribution of the data. For the same reason, the figures showing the results of the endurance test also present median values. Changes in EMG, relaxation, and contraction rates were analysed with repeated measures analysis of variance. $p$ Values $<0.05$ were regarded as statistically significant.

\section{ETHICS}

The study was conducted in accordance with the Helsinki Declaration II. Informed consent was obtained from all subjects. The study protocol was approved by the local ethics committee.

\section{Results}

Five of the patients with fibromyalgia but none of the controls were incapable of reaching a degree of central activation of at least $50 \%$. Thus the "true" muscle strength could not be reliably determined and they could not perform the endurance test. These patients were secondarily excluded. Without exclusion of these five patients the median degree of central activation was 0.66 in the patients compared with 0.79 in the controls $(p=0.07$, Mann-Whitney test). 
Basic measures of the 20 patients and 21 controls

\begin{tabular}{lcc}
\hline & Fibromyalgia & \multicolumn{1}{c}{ Controls } \\
\hline Midthigh: & & \\
Middle circumference (cm) & $55 \cdot 4(5 \cdot 4)$ & $57 \cdot 7(4 \cdot 8)$ \\
Fat thickness (cm) & $1 \cdot 6(0 \cdot 6)$ & $1 \cdot 6(0 \cdot 4)$ \\
Fat free area (cm $\left.{ }^{2}\right)$ & $164(30)$ & $181(29)$ \\
Voluntary strength (Nm) & $62(25)$ & $104(27)^{* * *}$ \\
Degree of activation (\%) & $75(4)$ & $79(6)$ \\
Physical activity (METs) & $34(5)$ & $41(6)^{* * *}$ \\
\hline$\star \star \star$ &
\end{tabular}

The age of the 20 participating patients with fibromyalgia was mean 48 (10) years as compared with 49 (6) years in the 21 controls. The weight and height were also similar in the two groups (61 (9) $\mathrm{kg} v 64$ (9) kg, and 164 (7) $\mathrm{cm} v 166$ (9) $\mathrm{cm}$ (all NS)).

The cross sectional fat free area of the thigh was not significantly less in the patient group (table). Maximal voluntary muscle strength was significantly lower in patients than in the control group, whereas the degree of central activation was similar. The estimated "true" muscle strength was 133 (28) Nm in the control group compared with 82 (26) $\mathrm{Nm}$ in the fibromyalgia group $(p<0.001)$. Muscle strength was related to fat free cross sectional area (fig 2). The mean strength was lower, being $0.50(0.15) \mathrm{Nm} / \mathrm{cm}^{2}$ in the patients with fibromyalgia compared with $0.74 \quad(0.15)$ $\mathrm{Nm} / \mathrm{cm}^{2}$ in the controls $(\mathrm{p}<0.001)$.

If subjects who performed less than $70 \%$ were excluded (see methods) similar results were obtained. The estimated degree of central activation was similar $(84.4(9) \%$ in these 12 patients with fibromyalgia $v 86.7$ (11)\% in 15 controls). The weights were also alike (61 $\mathrm{kg} v 63 \mathrm{~kg}$ ). The estimated "true" maximal contractile strength of the patients with fibromyalgia was 80 (24) $\mathrm{Nm} v 127$ (23) $\mathrm{Nm}$ in the controls $(p<0.001)$. The estimated "true" muscle strength per cross sectional area was lower $\left(0.46(0.09) \mathrm{Nm} / \mathrm{cm}^{2}\right.$ in the patients and $0.73(0.17) \mathrm{Nm} / \mathrm{cm}^{2}$ in the controls $(\mathrm{p}<0.001))$.

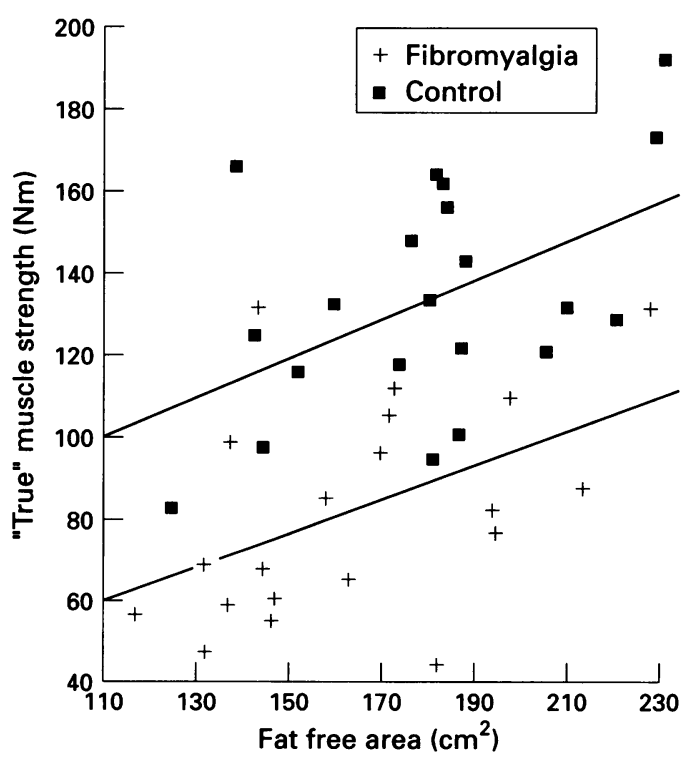

Figure 2 Relation between estimated "true" muscle strength and cross sectional fat free midthigh area. Linear regression lines for each group are shown.

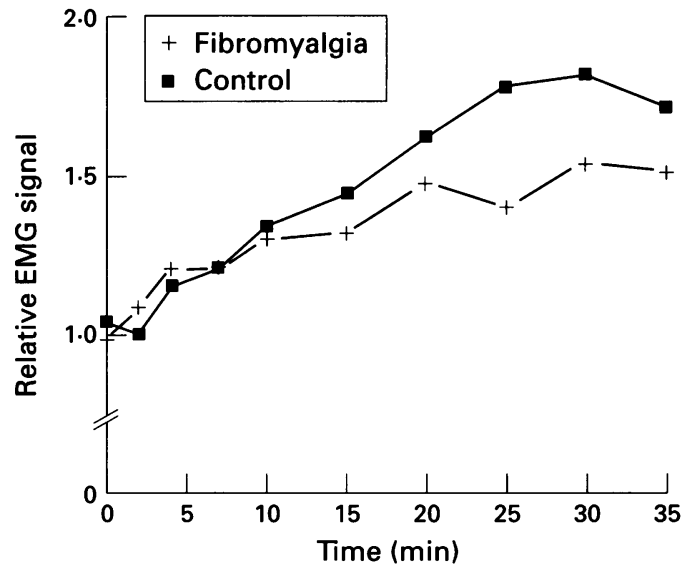

Figure 3 Relative mean EMG amplitude at a contraction level of $50 \%$ of maximum (median values).

The correlation between the degree of central activation and the estimated contractile strength per fat free area was low for the control group and the fibromyalgia group separately or together (all $R<0 \cdot 15, \mathrm{p}>0 \cdot 3$ ).

The reported physical activity level was lower in the patients with fibromyalgia (table), but the correlation between physical activity level and estimated "true" muscle strength per area was low $(R=0.26, \mathrm{p}=0.26$ in the control group and $R=0.00, \mathrm{p}=0.97$ in the fibromyalgia group).

\section{Endurance test}

The endurance time was median $22(13-37)$ minutes in the patients as compared with 29 (19-40) minutes in the control group (NS). Three of the patients and one control subject were unable to endure for a sufficiently long time to determine the exponential constant for the twitch decline at $50 \%$ of maximum. These subjects were excluded from further analysis.

The exponential constant was 1.68 $(1 \cdot 14-5 \cdot 5) \mathrm{h}^{-1}$ in the fibromyalgia group

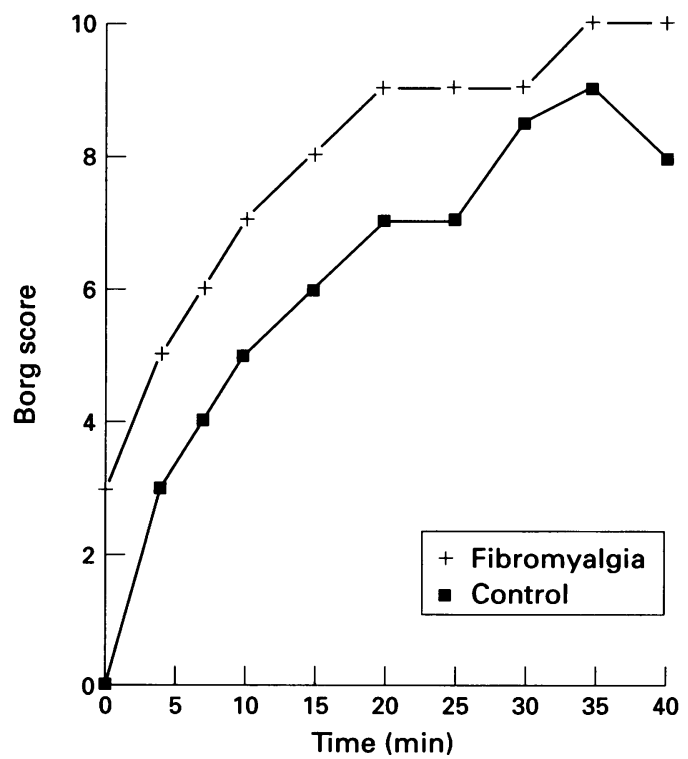

Figure 4 Borg scoring of perceived exertion in the two groups during the endurance test (median values). 


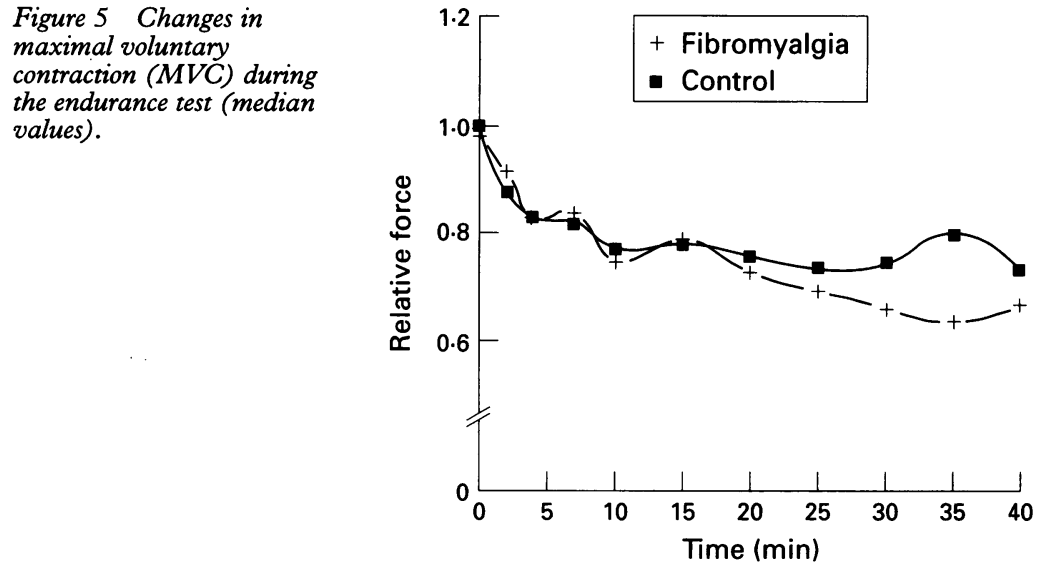

compared with $2.07(1.05-5.95) \mathrm{h}^{-1}$ in the control group (NS).

The increase in the relative mean EMG amplitude was similar in the two groups during the initial period (fig 3 ). There were no significant differences in increase in normalised contraction rate or relaxation rate over time. In all objective indices (twitch amplitude, EMG, contraction rate, relaxation rate) a non-significant trend towards a larger change in the control group could be observed. The patients scored higher on the Borg scale for perceived effort during the first 20 minutes of the test (fig 4). The decline in maximum voluntary contraction was not significantly different but there was a trend towards a faster decline in the patients with fibromyalgia (fig 5).

\section{Discussion}

Our study confirms previous findings of pronounced decreased voluntary muscle strength in the patients with fibromyalgia. A significant decrease of $30-40 \%$ in estimated "true" muscle strength per cross sectional area could also be seen in the patients, both with and without exclusion of those performing less than $70 \%$ of maximal activation.

In a methodological study, we found a slight underestimation of the contractile capacity if the subjects performed submaximally. As a similar degree of central activation was found in the two groups this does not explain the difference. Our inability to show any correlation between degree of activation and estimated contractile strength supports this. The degree of central activation in normal subjects $(79 \%)$ was lower in this study than previous reports. ${ }^{22} 23$ This might be because the digital averaging of the torque signals enabled us to register very small twitches, and partly because the control group consisted of sedentary middle aged women.

The patients were only measured with a $90^{\circ}$ flexion in the knee joint. We cannot exclude the possibility that a part of the difference was due to a difference between the two groups with respect to the angle of maximal isometric torque, but this is not likely. The measured torques may be lower due to excessive activity in the antagonistic muscles. A simultaneous EMG analysis of the antagonists might answer that question.

We were unable to match the control group completely with the very low physical activity of the patients. Pain and low physical activity for years might effect the muscles in a similar manner to immobilisation, which has been extensively investigated experimentally. Physical immobilisation is known to decrease voluntary muscle strength more than muscle area. ${ }^{24} 25$ This has been suggested to be caused partly by an increased amount of connective tissue and partly by a reduced neural drive; the second is adjusted for in our calculations. Previous studies have also suggested a larger decrease in wasting of the quadriceps than in other muscle groups after immobilisation. ${ }^{26}$

Patients with fibromyalgia might also show an altered central motor unit activation during work, causing secondary muscle damage, as hypothesised for occupational muscle pain. ${ }^{27}$

Our results are in contrast with studies of other muscle groups in patients with chronic fatigue syndrome or effort syndromes, where no reduction in muscle strength could be found.$^{28}$ The main symptoms in these conditions are fatigue, but muscle pain is commonly reported, and a large overlap has been reported with fibromyalgia. ${ }^{30}$ Although almost $90 \%$ of patients with fibromyalgia complain of severe fatigue only about $25 \%$ seem to fulfil proposed criteria for the chronic fatigue syndrome. $^{31}$

The voluntary endurance time was only slightly shorter in the fibromyalgia group possibly due to the selection of good performers. The patients with fibromyalgia in this study were selected only if they were known to cooperate fully with the muscle strength measurements, and the subjects who were not able to sustain a $50 \%$ contraction were secondarily excluded. The patients scored higher for perceived effort, however, than the controls.

The decline in the interpolated twitch sizes were similar in the two groups indicating no apparent failure of the excitation/contraction coupling in fibromyalgia.

The increase in EMG amplitude, contraction rate, and relaxation rate indices were also similar in the two groups. Our findings of normal changes in electrophysiological markers of fatigue in the patients with fibromyalgia are in accordance with studies showing normal ATP turnover in fibromyalgia using NMR spectroscopy, ${ }^{32} 33$ and with studies of other muscle groups in patients with chronic fatigue syndrome or effort syndromes. ${ }^{28} 29$

One could argue that the variation in the objective variables are so large that we may have overlooked small differences between the groups. As the trend in all objective variables, however, was towards larger changes within the control group, nothing points towards fibromyalgia being associated with any abnormality in the variables studied.

In conclusion, we found a reduced muscle strength per cross sectional area in the patients with fibromyalgia, implying that the patients do have impaired muscle function. 
Abnormalities in the basic energy metabolism of the muscles are, however, not assumed. This might indicate that the changes found are secondary to physical inactivity and altered central motor unit activation, maybe in combination with neuroendocrine factors. The reduced muscle strength confirms that exercise training should be an important part of the rehabilitation. ${ }^{34}$

We express our thanks to the Oak Foundation, Switzerland, the Danish Rheumatism Association, and the Bodil Petersen Foundation for their support of the study. We are also indebted to Jesper Mehisen MD for help in the planning of the study and for revision of the manuscript.

1 Wolfe F, Smythe HA, Yunus MB, et al. The American College of Rheumatology 1990 criteria for the classification of fibromyalgia. Report of the Multicenter Criteria tion of fibromyalgia. Report of the Multicenter

2 Yunus MB, Kalyan Raman UP. Muscle biopsy findings in primary fibromyalgia and other forms of nonarticular primary fibromyalgia and other forms of nonarticular
rheumatism. Rheum Dis Clin North Am 1989;15:115-34.

3 Schroder HD, Drewes AM, Andreasen A. Muscle biopsy in fibromyalgia. F Musculoskeletal Pain 1993;1:165-9.

4 Bengtsson A, Henriksson KG, Larsson J. Reduced highenergy phosphate levels in the painful muscles of patients with primary fibromyalgia. Arthritis Rheum 1986;29:817-21.

5 Lund N, Bengtsson A, Thorborg P. Muscle tissue oxygen pressure in primary fibromyalgia. Scand $\mathcal{f}$ Rheumatol 1986;15:165-73.

6 Jacobsen S, Danneskiold-Samsøe B. Isometric and isokinetic muscle strength in patients with fibrositis syndrome. New characteristics for a difficult definable category of patients. Scand $\mathcal{F}$ Rheumatol 1987;16:61-5.

7 Jacobsen S, Danneskiold Samsøe B. Dynamic muscular endurance in primary fibromyalgia compared with chronic myofascial pain syndrome. Arch Phys Med Chronic myofascial pail 1992;73:170-3.

8 Bengtsson A, Henriksson KG. The muscle in fibromyalgia - a review of Swedish studies. $\mathcal{F}$ Rheumatol Suppl 1989;19:144-9.

9 Merton PA. Voluntary strength and fatigue. I Physiol (Lond) 1954;123:553-64

10 Hales JP, Gandevia SC. Assessment of maximal voluntary contraction with twitch interpolation: an instrument to measure twitch responses. $\mathscr{f}$ Neurosci Meth 1988;25: 97-102.

11 Rutherford OM, Jones DA, Newham DJ. Clinical and experimental application of the percutaneous twitch superimposition technique for the study of human muscle activation. $f$ Neurol Neurosurg Psychiatry 1986;49: 1288-91.

12 Bigland-Ritchie B, Furbush F, Woods JJ. Fatigue of intermittent submaximal voluntary contractions: central and mittent submaximal voluntary contractions: cen

13 Edwards RHT, Hill DK, Jones DA. Fatigue of long duration in human skeletal muscle after exercise. $¥$ Physiol tion in human skeletal mis

14 Sandercock TG, Faulkner JA, Albers JW, Abbrecht PH. Single motor unit and fiber action potentials during fatigue. Eur F Appl Physiol 1985;58:1073-9.

15 Sallis JF, Haskell WL, Fortmann SP, Rogers T, Blair SN, Paffenbarger RS, Jr. Physical activity assessment methodology in the five city project. Am $\mathcal{F}$ Epidemiol 1985;121:91-106.

16 Booth RAD, Goddard BA, Paton A. Measurement of fat thickness in man: a comparison of ultrasound, harpenden calibers and electrical conductivity. $\mathrm{Br} F \mathrm{Nutr}$ 1966;20:719-25.

17 Heckmatt JZ, Pier N, Dubowitz V. Measurement of quadriceps muscle thickness and subcutaneous tissue thickness in normal children by real time ultrasound imaging. $f$ Clin Ultrasound 1988;16:171-6.

18 Bülow PM, Nørregaard J, Danneskiold-Samsøe B Mehlsen J. The twitch interpolation technique in testing of maximal muscle strength: influence of potentiation force level, stimulus intensity and preload. Eur $\mathfrak{F} A p p$ force level, stimulus inter
Physiol 1993;67:462-6.

19 Bigland-Ritchie B, Cafarelli E, Vøllestad NK. Fatigue of submaximal static contractions. Acta Physiol Scand 1986;128: $137-48$.

20 Thomas CK, Johansson RS, Westling G, Bigland-Ritchie B. Twitch properties of human thenar motor units measured in response to interneural motor-axon stimulation. f Neurophysiol 1990;64:1339-46.

21 Borg G. A category scale with ratio properties for intermodal and interindividual comparisons. In: Geissler $\mathrm{H}$, et al, eds. Psychological judgment and the process of perception. Amsterdam: North Holland, 1982:25-34.

22 Belanger AY, McComas AJ. Extent of motor unit activation during effort. $₹$ Appl Physiol 1981;51:1131-5.

23 Chapman SJ, Edwards RHT, Greig C, Rutherford O Practical application of the twitch interpolation technique for the study of voluntary contration of the quadriceps muscle in man. F Physiol (Lond) 1984;353:3P

24 Appell HJ. Muscular atrophy following immobilisation. A review. Sports Med 1990;10:42-58.

25 Kannus $P$, Jozca L, Renström P, et al. The effects of training, immobilisation and remobilisation on musculoskeletal tissue. Scand ₹ Med Sci Sports 1992;2:100-18.

26 Sargeant AJ, Davies CTM, Edwards RHT, Maunder C, Young A. Functional and structural changes after disuse of human muscle. Clin Sci Mol Med 1977;52:337-42.

27 Edwards RHT. Hypotheses of peripheral and central mechanisms underlying occupational muscle pain and injury. Eur 7 Appl Physiol 1988;57:275-81.

28 Lloyd AR, Gandevia SC, Hales JP. Muscle performance, voluntary activation, twitch properties and perceived voluntary activation, twitch properties and perceived
effort in normal subjects and patients with the chronic fatigue syndrome. Brain 1991;114:85-98.

29 Stokes MJ, Cooper RG, Edwards RHT. Normal muscle strength and fatigability in patients with effort syndromes. $B M F$ 1988;297:1014-7.

30 Goldenberg DL, Simms RW, Geiger A, Komaroff AL High frequency of fibromyalgia in patients with chronic fatigue seen in a primary care practice. Arthritis Rheum 1990;33:381-7.

31 Nørregaard J, Bülow PM, Prescott E, Jacobsen S, Danneskiold-Samsøe B. A four-year follow-up study in fibromyalgia. Relationship to chronic fatigue syndrome. Scand $₹$ Rheumatol 1993;22:35-8.

32 de Blecourt AC, Wolf RF, van Rijswijk MH, Kamman RL, Knipping AA, Mooyaart EL. In vivo $31 \mathrm{P}$ magnetic resonance spectroscopy (MRS) of tender points in patients with primary fibromyalgia syndrome. Rheumatol Int with primary
$1991 ; 11: 51-4$.

33 Jacobsen S, Jensen KE, Thomsen C, Danneskiold-Samsøe $\mathrm{B}$, Henriksen $\mathrm{O}$. $31 \mathrm{P}$ magnetic resonance spectroscopy
of skeletal muscle in patients with fibromyalgia. of skeletal muscle in pa

34 McCain GA, Bell DA, Mai FM, Halliday PD. A controlled study of the effects of a supervised cardiovascular fitness training program on the manifestations of primary fibromyalgia. Arthritis Rheum 1988;31:1135-41. 